\title{
Proposta metodológica para previsão de impactos decorrentes de acidentes com barragens de rejeito
}

\author{
Methodological proposal to predict impacts arising \\ from accidents with tailings dams
}

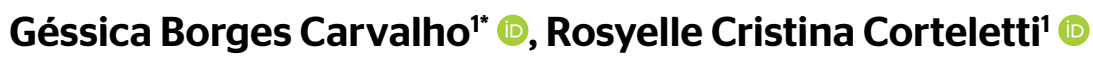

\section{RESUMO}

O rompimento de barragens de rejeitos é alvo de preocupação em função do alto dano potencial associado, sendo necessária uma avaliação de previsão de impactos que permita melhor caracterização da área a jusante, e que a coleta de dados seja realizada pelos órgãos fiscalizadores, e não por parte dos empreendedores. Este trabalho teve por objetivo propor uma classificação dos impactos decorrentes de rompimentos desses barramentos, tendo como referência as avaliações de danos adotadas pelas legislações de segurança de barragens no Brasil. Para isso, foi elaborada uma tabela de critérios e subcritérios com os parâmetros que influenciam na determinação dos impactos causados, os quais foram hierarquizados por meio da aplicação do Analytic Hierarchy Process (AHP), proposto por Saaty (1991). Para determinação dos valores de importância de cada impacto, utilizou-se a técnica $A d-H o c$ por meio da dinâmica do brainstorming, com profissionais que realizaram julgamentos a partir de comparações paritárias dos critérios adotados. A ocupação humana na área a jusante, como observado nas legislações, apresentou o maior peso. O volume do reservatório e os impactos no meio socioeconômico apresentaram pesos inferiores aos impactos nos meios físico e biótico, contrastando com as legislações. A aplicação da metodologia em barragem do Quadrilátero Ferrífero comprovou a coerência do índice de impactos ambientais (IA) proposto, indicando as fragilidades da área atingida. O detalhamento da área a jusante permitiu identificar aspectos que podem potencializar ou amortizar os impactos, como periculosidade do rejeito, volume do reservatório e proximidade de centros urbanos.

Palavras-chave: barragens de rejeitos; avaliação de impactos; analytic hierarchy process.

\begin{abstract}
The tailings dam failures is a matter of concern due to the high damage potential associated, requiring an assessment of forecast impacts that allows better characterization of downstream area, and that data collection is performed by regulatory agencies and not by the entrepreneurs. This work aimed to propose a classification of impacts resulting from hypothetical tailings dams failure, based on the damage assessment adopted by dam safety legislation in Brazil. For that, a table of criteria and subcriteria was elaborated with the parameters that influence the determination of the resulting impacts, which were hierarchized through the application of the Analytic Hierarchy Process (AHP), proposed by Saaty (1991). To determine the weights of each impact, the Ad-Hoc technique was used through the dynamics of brainstorming. with professionals who made judgments from parity comparisons of the determined criteria. Human occupation in the downstream area, as noted in the legislation, presented the greatest weight. The volume of the reservoir and the impacts on the socioeconomic environment presented lower weights to the impacts in the physical and biotic environments, in contrast with the legislation. The application of the methodology in the Quadrilatero Ferrifero dam proved the consistency of the proposed impact index (IA), indicating the fragilities of the affected area. The detailing of the downstream area allowed to identify aspects that could potentiate or amortize the impacts, such as hazardous waste, reservoir volume, and proximity to urban centers.
\end{abstract}

Keywords: waste dams; impact assessment; analytic hierarchy process.

\section{INTRODUÇÃO}

O Brasil é um país historicamente marcado pela intensa exploração das suas riquezas minerais. A atividade mineradora tornou-se um importante segmento da sua economia, tendo papel significativo na produção de bens e serviços, no desenvolvimento e na contribuição para a melhoria do bem-estar e da qualidade de vida de sua população. Em contrapartida, é responsável por grandes impactos ambientais, seja na área da mina, seja na disposição do material estéril e dos rejeitos que são gerados no processo de beneficiamento.

'Núcleo de Geotecnia da Escola de Minas, Universidade Federal de Ouro Preto - Ouro Preto (MG), Brasil.

*Autora correspondente: gessicaborgescarvalho@gmail.com

Conflitos de interesse: os autores declaram não haver conflito de interesses.

Financiamento: nenhum.

Recebido: 22/02/2019 - Aceito: 02/06/2020 - Reg. ABES: 20190061 
O método mais utilizado para a disposição dos rejeitos são os diques e as barragens. De acordo com o Inventário de Barragens publicado pela Fundação Estadual do Meio Ambiente (FEAM), estão cadastradas, no Banco de Declarações Ambientais (BDA), 698 estruturas em Minas Gerais, sendo $62,3 \%$ do total de cadastros referentes a barragens de contenção de rejeitos da mineração (FEAM, 2017).

Os rompimentos de barragens são eventos de alto dano potencial. Como exemplo, tem-se a barragem de Fundão, empreendimento da Samarco Mineração S.A., localizada em Bento Rodrigues, subdistrito de Mariana, Minas Gerais. O rompimento, ocorrido em 5 de dezembro de 2015, representou o maior desastre ambiental do Brasil e o maior do mundo com barragens de rejeito. Entre os impactos, pode-se citar: contaminação dos rios Gualaxo do Norte, do Carmo e Doce, tendo chegado ao Espírito Santo e atingido o Oceano Atlântico; 19 mortes; desalojamento de populações; interrupção de serviços como água e energia elétrica; e contaminação da fauna e da flora (IBAMA, 2015; FEAM, 2016).

Em 25 de janeiro de 2019, ocorreu o rompimento da barragem B1 de contenção de rejeitos de minério de ferro, pertencente ao Complexo da Mina do Córrego do Feijão, localizada em Brumadinho, Minas Gerais. O empreendimento da Vale S.A está inserido na Bacia do Rio São Francisco. Os rejeitos atingiram duas outras barragens, alcançou o rio Paraopeba e ocasionou centenas de vítimas, ultrapassando o dano de vidas humanas registradas pelo rompimento da barragem de Fundão. Os impactos ambientais ainda estão sendo mensurados (FEAM, 2019).

De forma a avaliar o risco e o dano potencial das barragens de contenção de rejeitos, anualmente, são divulgados os inventários de barragens pela FEAM e a classificação das barragens de mineração pelo extinto Departamento Nacional de Produção Mineral (DNPM), atual Agência Nacional da Mineração (ANM). Para essas classificações, são utilizadas, principalmente, a Deliberação Normativa do Conselho Estadual de Política Ambiental (DN COPAM) nº 87/2005 (COPAM, 2005) e a Portaria DNPM no 70.389/2017 (DNPM, 2017).

De acordo com a classificação de barragens de mineração divulgada pelo então extinto DNPM em dezembro de 2016, das 447 barragens cadastradas e inseridas na Lei Federal no 12.334/2010 (BRASIL, 2010), 223 são consideradas de alto dano potencial. Apesar disso, não se pode afirmar que as 223 barragens impactarão o ambiente nas mesmas intensidade e magnitude, como pode ser avaliado pelos exemplos citados anteriormente.

Nesse contexto, o trabalho teve como objetivo realizar uma análise crítica das legislações vigentes voltadas para a classificação de barragens de rejeito, de forma a propor uma metodologia de classificação dos impactos decorrentes de rupturas desses reservatórios. Tais impactos serão quantificados e hierarquizados por meio da utilização dos Métodos Multicritérios de Apoio à Decisão (MCDA), subsidiados no Analytic Hierarchy Process (AHP), proposto por Saaty (1991).

Além disso, deve-se ressaltar o crescimento da aplicação da análise multivariada em trabalhos que se referem à identificação de impactos, à fragilidade ambiental, à análise de risco e à classificação de perigo, como observado em pesquisas realizadas por Bernardi, Fowler e Landim (2001), Brito et al. (2006), Denúbila (2013), Gimenes e Filho (2013), Corteletti (2014) e Donassollo (2017). Entretanto, há uma carência em dados e pesquisas referentes ao uso do AHP na avaliação e na previsão dos impactos oriundos da mineração, especialmente com barragens de contenção de rejeitos. Dessa forma, este trabalho buscou servir como fonte de dados a respeito da aplicabilidade e da consistência do método para essa finalidade.

\section{METODOLOGIA}

Os procedimentos metodológicos foram realizados em três etapas, as quais são apresentadas na Figura 1.

\section{Definição dos critérios para avaliação de impactos}

A definição dos critérios para previsão dos impactos ambientais em casos de rompimentos de barragens de contenção de rejeitos teve como pilar o levantamento de dados a respeito das legislações ambientais, das portarias e das normativas relacionadas à segurança de barragens e os estudos referentes aos impactos ambientais ocasionados por eventos iguais ou semelhantes.

Para a classificação das barragens por dano potencial associado (DPA), a Portaria DNPM n ${ }^{\circ} 70.389 / 2017$, assim como a Resolução do Conselho Nacional de Recursos Hídricos (CNRH) nº 143/2012, consideram como critérios os seguintes aspectos: volume total do reservatório, existência de população a jusante, impacto ambiental e impacto socioeconômico (CNRH, 2012; DNPM, 2017).

A DN COPAM n 87/2005, que atualizou e complementou a DN COPAM n ${ }^{\circ}$ 62/2002, considera para o cálculo do potencial de dano ambiental, além dos critérios já mencionados, a altura da barragem (COPAM, 2005). De modo a identificar as fragilidades existentes nessas normas, foi elaborado o Quadro 1, com o objetivo de realizar um comparativo entre as classificações existentes no estado de Minas Gerais e no Brasil.

Por meio do Quadro 1, pode-se observar que a Resolução do CNRH n ${ }^{\circ}$ 143/2012 e a Portaria DNPM no 70.389/2017 atribuem os maiores pesos para a ocupação humana a jusante. Em segundo lugar, estão os impactos ambientais. Dessa forma, esses dois critérios foram considerados imprescindíveis para a análise dos impactos de rompimentos de barragens de contenção de rejeitos.

O terceiro critério definido para a avaliação do índice dos impactos previstos foi o volume do reservatório, uma vez que é comum em todas as legislações analisadas no Quadro 1. A presença desse critério se justifica em razão de o volume de rejeito armazenado ser fator diretamente influente no grau dos impactos. Pressupõe-se que, quanto maior o volume do reservatório, maior a quantidade de rejeito eventualmente liberada e, consequentemente, maiores serão o volume de material deslocado e os impactos na área a jusante da barragem.

Com os três critérios definidos, foi realizada uma análise da Resolução do Conselho Nacional de Meio Ambiente (CONAMA) nº 001/1986 a respeito do conceito de impacto ambiental. De acordo com a resolução, é considerado impacto ambiental: alterações que afetem a saúde, a segurança e o bem-estar da população; as atividades socioeconômicas; a biota; as condições estéticas e sanitárias do meio ambiente; e a qualidade dos recursos naturais (BRASIL, 1986). Objetivando melhor análise dos impactos ambientais, esse critério foi dividido em outros três, a saber: impactos no meio físico, impactos no meio biótico e impactos socioeconômicos.

\begin{tabular}{|c|c|c|}
\hline $\begin{array}{c}\text { 1a etapa: } \\
\text { definição dos } \\
\text { critérios para a } \\
\text { nova avaliação de } \\
\text { impactos }\end{array}$ & $\begin{array}{l}\text { 2a etapa: } \\
\text { realização do } \\
\text { brainstorming } \\
\text { e aplicação do } \\
\text { método AHP }\end{array}$ & $\begin{array}{c}\text { 3a etapa: } \\
\text { elaboração da } \\
\text { metodologia para } \\
\text { a proposta de } \\
\text { classificação das } \\
\text { barragens quanto } \\
\text { aos impactos } \\
\text { e aplicação da } \\
\text { metodologia } \\
\text { proposta }\end{array}$ \\
\hline
\end{tabular}

Figura 1 - Fluxograma das etapas metodológicas. 
Quadro 1 - Classificação das barragens de rejeito em função do dano potencial associado de acordo com as legislações vigentes em âmbitos nacional e estadual.

\begin{tabular}{|c|c|c|c|c|c|}
\hline \multicolumn{6}{|c|}{ Volume do reservatório $\left(\times 106 \mathrm{~m}^{3}\right)$} \\
\hline $\begin{array}{c}\text { Resolução CNRH } \\
\text { no 143/2012 }\end{array}$ & $\begin{array}{c}\mathrm{Vr} \leq 0,5 \\
\quad(1)\end{array}$ & $\begin{array}{c}0,5<V_{r} \leq 5 \\
\text { (2) }\end{array}$ & $\begin{array}{c}5<\operatorname{Vr} \leq 25 \\
\text { (3) }\end{array}$ & $\begin{array}{c}25<V_{r}<50 \\
\text { (4) }\end{array}$ & $\begin{array}{l}\mathrm{V} r \geq 50 \\
\quad(5)\end{array}$ \\
\hline $\begin{array}{l}\text { Portaria DNPM } \\
\text { no 70.389/2017 }\end{array}$ & $\begin{array}{c}V_{r} \leq 0,5 \\
\quad(1)\end{array}$ & $\begin{array}{c}0,5<V_{r} \leq 5 \\
\text { (2) }\end{array}$ & $\begin{array}{c}5<V r \leq 25 \\
\text { (3) }\end{array}$ & $\begin{array}{c}25<V_{r}<50 \\
\text { (4) }\end{array}$ & $\begin{array}{l}\mathrm{Vr} \geq 50 \\
\quad(5)\end{array}$ \\
\hline $\begin{array}{l}\text { DN COPAM } \\
\text { no 87/2005 }\end{array}$ & $\begin{aligned} V r & <0,5 \\
V & =0\end{aligned}$ & $\begin{array}{c}0,5<V_{r} \leq 5 \\
V=1\end{array}$ & $\begin{array}{l}V r>5 \\
V=2\end{array}$ & - & - \\
\hline \multicolumn{6}{|c|}{ Altura da barragem $(\mathrm{m})$} \\
\hline $\begin{array}{l}\text { DN COPAM } \\
\text { no 87/2005 }\end{array}$ & $\begin{array}{l}H<15 \\
V=0\end{array}$ & $\begin{array}{c}15 \leq H \leq 30 \\
V=1\end{array}$ & $\begin{array}{l}H>30 \\
V=2\end{array}$ & - & - \\
\hline \multicolumn{6}{|c|}{ Ocupação humana a jusante } \\
\hline $\begin{array}{c}\text { Resolução CNRH } \\
\text { no 143/2012 }\end{array}$ & $\begin{array}{c}1 \\
(0)\end{array}$ & $\begin{array}{l}\text { PF } \\
\text { (3) }\end{array}$ & $\begin{array}{l}F \\
(5)\end{array}$ & $\begin{array}{l}\text { Ex } \\
(10)\end{array}$ & - \\
\hline $\begin{array}{l}\text { Portaria DNPM } \\
\text { no 70.389/2017 }\end{array}$ & $\begin{array}{c}1 \\
(0)\end{array}$ & $\begin{array}{l}\mathrm{PF} \\
(3)\end{array}$ & $\begin{array}{l}F \\
(5) \\
\end{array}$ & $\begin{array}{l}\text { Ex } \\
(10) \\
\end{array}$ & - \\
\hline $\begin{array}{l}\text { DN COPAM } \\
\text { no 87/2005 }\end{array}$ & $\begin{array}{c}1 \\
v=0\end{array}$ & $\begin{array}{c}E v \\
V=2\end{array}$ & $\begin{array}{c}E x \\
v=3\end{array}$ & $\begin{array}{c}G \\
V=4\end{array}$ & - \\
\hline \multicolumn{6}{|c|}{ Impactos ambientais/interesse ambiental a jusante } \\
\hline $\begin{array}{c}\text { Resolução CNRH } \\
\text { no 143/2012 }\end{array}$ & $\begin{array}{l}\text { In } \\
(0)\end{array}$ & $\begin{array}{l}\text { PS } \\
\text { (2) }\end{array}$ & $\begin{array}{c}S \\
(6) \\
\end{array}$ & $\begin{array}{l}\text { MS } \\
(8)\end{array}$ & $\begin{array}{l}\text { MSA } \\
(10)\end{array}$ \\
\hline $\begin{array}{l}\text { Portaria DNPM } \\
\text { no 70.389/2017 }\end{array}$ & $\begin{array}{l}\text { In } \\
(0)\end{array}$ & $\begin{array}{l}\text { PS } \\
\text { (2) }\end{array}$ & $\begin{array}{c}S \\
(6)\end{array}$ & $\begin{array}{l}\text { MS } \\
(8)\end{array}$ & $\begin{array}{l}\text { MSA } \\
(10)\end{array}$ \\
\hline $\begin{array}{l}\text { DN COPAM } \\
\text { no 87/2005 }\end{array}$ & $\begin{array}{c}P S \\
V=0\end{array}$ & $\begin{array}{c}S \\
V=2\end{array}$ & $\begin{array}{c}E \\
V=3\end{array}$ & - & - \\
\hline \multicolumn{6}{|c|}{ Impactos socioeconômicos/instalações na área a jusante } \\
\hline $\begin{array}{c}\text { Resolução CNRH } \\
\text { no 143/2012 }\end{array}$ & $\begin{array}{l}\text { In } \\
(0)\end{array}$ & $\begin{array}{l}\mathrm{B} \\
(1) \\
\end{array}$ & $\begin{array}{l}M \\
(3)\end{array}$ & $\begin{array}{c}\text { A } \\
(5)\end{array}$ & - \\
\hline $\begin{array}{l}\text { Portaria DNPM } \\
\text { no 70.389/2017 }\end{array}$ & $\begin{array}{l}\text { In } \\
(0)\end{array}$ & $\begin{array}{l}B \\
(1)\end{array}$ & $\begin{array}{l}M \\
\text { (3) }\end{array}$ & $\begin{array}{c}\text { A } \\
(5)\end{array}$ & - \\
\hline $\begin{array}{l}\text { DN COPAM } \\
\text { no 87/2005 }\end{array}$ & $\begin{array}{c}1 \\
v=0\end{array}$ & $\begin{array}{c}B \\
V=1\end{array}$ & $\begin{array}{c}A \\
V=2\end{array}$ & - & - \\
\hline
\end{tabular}

Fonte: CNRH (2012); COPAM (2005); DNPM (2017).

CNRH: Conselho Nacional de Recursos Hídricos; DNPM: Departamento Nacional de Produção Mineral; DN: Deliberação Normativa; COPAM: Conselho Estadual de Política Ambiental; Vr: volume do reservatório; V: valor do parâmetro; H: altura; I: inexistente; PF: pouco frequente; F: frequente; Ex: existente; Ev: eventual; G: grande; In: insignificante; PS: pouco significativo; S: significativo; MS: muito significativo; MSA: muito significativo agravado; E: elevado; B: baixo; M: médio; A: alto.

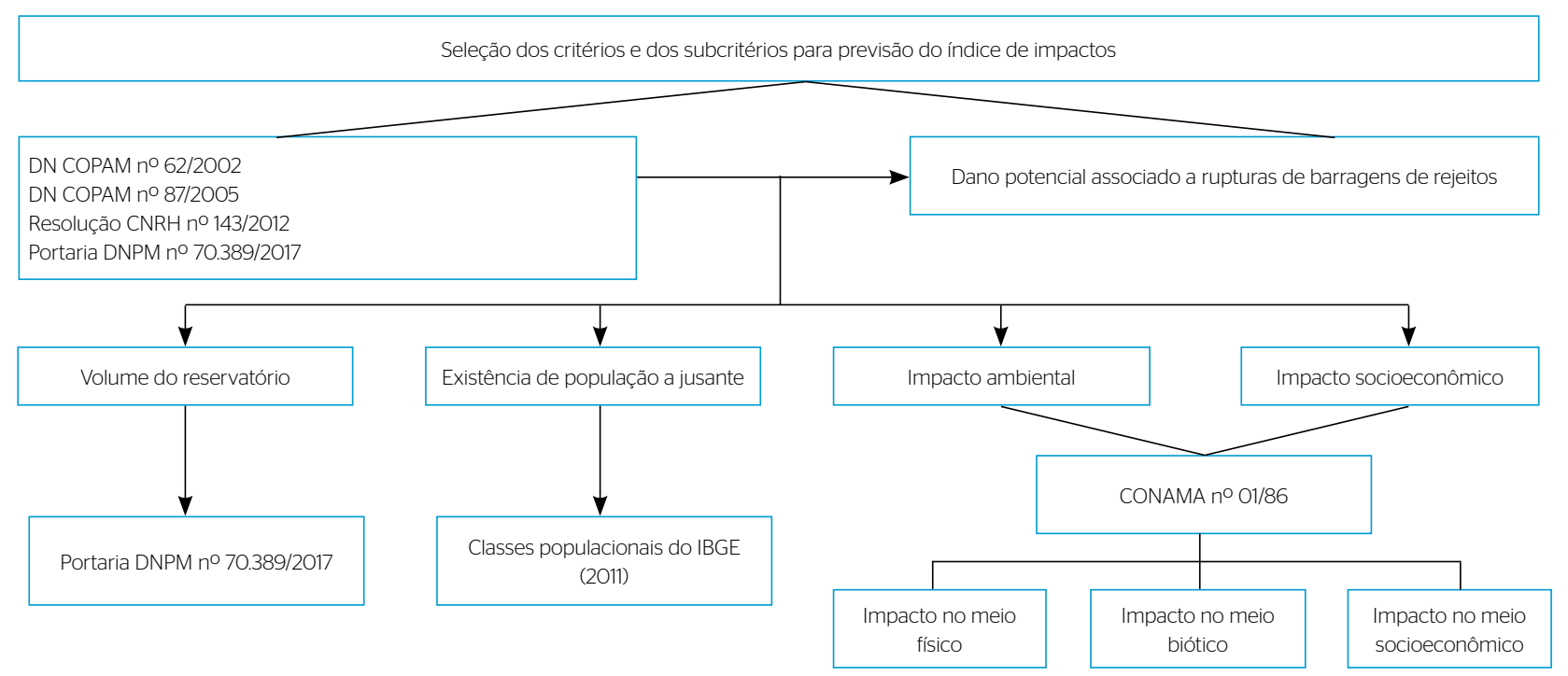

DN: Deliberação Normativa; COPAM: Conselho Estadual de Política Ambiental; CNRH: Conselho Nacional de Recursos Hídricos; DNPM: Departamento Nacional de Produção Mineral; CONAMA: Conselho Nacional do Meio Ambiente; IBGE: Instituto Brasileiro de Geografia e Estatística.

Figura 2 - Fluxograma da seleção de critérios e subcritérios para previsão de índice de impactos. 
Desse modo, a Figura 2 apresenta os critérios e os subcritérios definidos para a mensuração do índice de impactos previstos em casos de rompimentos de barragens.

Na prática do AHP, todos os critérios precisam de um total fixo de subcritérios. Nesse caso, foi determinado um total de cinco subcritérios para cada um. A definição dos subcritérios, destacados na Figura 2, estão descritos a seguir:

- Volume do reservatório: os subcritérios mantiveram-se iguais aos definidos na Portaria DNPM no 70.389/2017, descritos no Quadro 1;

- Existência de população a jusante: para estabelecer as cinco faixas populacionais, foram utilizadas as classes populacionais definidas pelo Instituto Brasileiro de Geografia e Estatística - IBGE (2011) na publicação Indicadores Sociais Municipais: Uma Análise dos Resultados do Universo do Censo Demográfico 2010;

- Impacto ambiental no meio físico: definido de acordo com o que é cobrado na elaboração dos estudos de impactos ambientais (EIAs) pela resolução CONAMA n ${ }^{\circ}$ 001/86, para análise do meio físico da região do empreendimento;

- Impacto ambiental no meio biótico: seguiu os mesmos critérios da análise dos impactos no meio físico, por meio do que é citado no art. $6^{\circ}$ da Resolução CONAMA no 001/86, em relação ao meio biótico;

- Impacto ambiental no meio socioeconômico: definido de acordo com o art. $6^{\circ}$ da Resolução CONAMA nº 001/86, para o meio socioeconômico.

\section{Brainstorming e aplicação do método Analytic Hierarchy Process}

O método AHP, proposto por Saaty (1991), foi utilizado para quantificação dos critérios considerados para avaliar os impactos decorrentes de um rompimento hipotético de uma barragem e a comparação com os pesos definidos nas normas e legislações vigentes. Dessa forma, é necessária a realização da comparação paritária entre os critérios definidos.

Segundo Saaty (1991), essa é uma maneira racional de lidar com os julgamentos, uma vez que, por meio da comparação por pares, as prioridades calculadas pelo AHP permitem capturar medidas subjetivas e objetivas, demonstrando o domínio de um critério sobre o outro e de uma alternativa sobre a outra, determinando um ranking de alternativas.

Para aplicação do brainstorming, foi elaborada uma tabela composta da coluna X e da linha Y (Tabela 1), para comparação paritária dos critérios definidos no item anterior.

A realização do brainstorming contou com a participação de sete especialistas com formações complementares da área geotécnica: três engenheiros civis, duas engenheiras ambientais, um engenheiro de minas e um geólogo. Inicialmente, foi entregue aos especialistas a Tabela 1 e realizada a seguinte pergunta: "Quanto o critério da coluna X é mais importante (relevante) que o critério da linha $\mathrm{Y}$, em relação aos impactos característicos do rompimento hipotético de uma barragem de contenção de rejeitos da mineração?”. Para responder a essa pergunta, os especialistas tiveram acesso à escala proposta por Saaty (1991), na Tabela 2.

Para estabelecer os pesos finais, seguiu-se a metodologia adotada por Donassollo (2017), a qual respeita as seguintes condições:

- Julgamentos com valores semelhantes devem ser mantidos;

- Julgamentos que destoam entre si devem ser discutidos entre os envolvidos por aproximadamente cinco minutos a fim de se alcançar um consenso no valor final;

- Casos em que não é possível encontrar um valor comum, o moderador é responsável por determinar o valor com base na discussão realizada sobre os critérios envolvidos.
Com os valores determinados no brainstorming, obteve-se uma matriz que permitiu comparar todos os elementos envolvidos na análise. Finalmente, a partir da matriz de comparação paritária, foram realizados os cálculos determinados pelo método AHP. Os passos realizados estão descritos a seguir (COSTA, 2002; DONASSOLLO, 2017):

Obteve-se a matriz normalizada a partir da matriz de comparação paritária, utilizando-se a Equação 1:

$A^{\prime}=\left[a_{i j}^{\prime}\right]$

Em que:

$\mathrm{a}^{\prime}=\frac{\mathrm{a}_{\mathrm{ij}}}{\sum_{\mathrm{k}=1}^{\mathrm{n}} \mathrm{a}_{\mathrm{ik}}}$, para $1 \leq i \leq n$ e $1 \leq j \leq n$.

Realizou-se o cálculo das prioridades médias locais (PML) por meio do valor médio obtido em cada linha da matriz normalizada, conforme a Equação 2.

$\mathrm{W}=\mathrm{W}_{\mathrm{k}}$

Em que:

$\mathrm{W}_{\mathrm{k}}=\frac{\sum_{\mathrm{i}=1}^{\mathrm{n}} \mathrm{a}{ }_{\mathrm{ij}}}{\mathrm{n}}$, para $1 \leq j \leq n$ e $1 \leq k \leq n$.

Realizou-se a avaliação da análise de consistência dos resultados, com o objetivo de mensurar o grau de inconsistência de uma matriz de julgamentos

Tabela 1 - Matriz quadrada para julgamento paritário dos critérios definidos para avaliação do impacto ambiental em caso de rompimento de barragens de contenção de rejeitos da mineração.

\begin{tabular}{|c|c|c|c|c|c|}
\hline Coluna X & 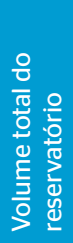 & 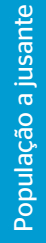 & 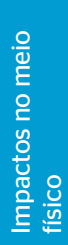 & 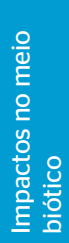 & 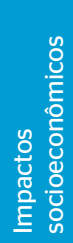 \\
\hline \multicolumn{6}{|l|}{ Volume total do reservatório } \\
\hline \multicolumn{6}{|l|}{ População a jusante } \\
\hline \multicolumn{6}{|l|}{ Impactos no meio físico } \\
\hline \multicolumn{6}{|l|}{ Impactos no meio biótico } \\
\hline Impactos socioeconômicos & & & & & \\
\hline
\end{tabular}

Tabela 2 - Escala fundamental de Saaty.

\begin{tabular}{c|c} 
Escala numérica & Escala verbal \\
\hline 1 & Mesma importância \\
\hline 3 & Importância moderada de um sobre o outro \\
\hline 5 & Importância essencial ou forte de um sobre o outro \\
\hline 7 & Importância muito forte \\
\hline 9 & Importância extrema \\
\hline $2,6,4,8$ & Valores intermediários \\
\hline
\end{tabular}

Fonte: Saaty (1991). 
paritários. Para obtenção da taxa de consistência (C), foram considerados o máximo autovalor e o tamanho da matriz, obtendo-se o índice de consistência (IC), seguindo-se as seguintes etapas:

- Calculou-se o vetor [C] por meio da multiplicação da matriz [I] pelo vetor coluna de pesos relativos [Wi], como mostra a Equação 3.

$[\mathrm{C}]=[\mathrm{I}] \cdot\left[\mathrm{W}_{\mathrm{i}}\right]$

- Posteriormente, obteve-se o vetor [D], o qual corresponde ao máximo autovalor da matriz [I], denotado por $\lambda$ Máx. Para tal, utilizaram-se as Equações 4 e 5 .

$D=\sum_{i=1}^{n} \frac{c_{i j}}{w_{i j}}$

$\lambda$ Máx $=\frac{[D]}{n}$

Em que:

$\mathrm{n}=$ ordem da matriz paritária de julgamentos.

- Realizou-se o cálculo do IC a partir do máximo autovalor da matriz [I], do $\lambda$ Max e da ordem da matriz paritária de julgamentos (n), que, nesse caso, é equivalente a 5. Para tal, foi utilizada a Equação 6. O valor de IC é considerado aceitável quando o valor obtido for menor que 0,1 .

$\underline{\mathrm{IC}=\lambda \mathrm{Máx}-\mathrm{n}}$

$\mathrm{n}-1$

- Calculou-se a razão de consistência (RC) para a avaliação da inconsistência em função da ordem da matriz de julgamentos, conforme a Equação 7.

$\mathrm{RC}=\mathrm{IC} / \mathrm{IR}$

O valor do índice randômico médio (IR) para uma matriz de ordem 5 foi verificado na Tabela 3, elaborada por Saaty (1991). Nesse caso, IR correspondeu a 1,12. O valor foi considerado aceitável quando $\mathrm{RC}<0,1$.

Dessa forma, com os valores de IC e RC aceitáveis, a próxima etapa consistiu na obtenção dos pesos para cada critério por meio dos valores determinados pelo vetor $\left[\mathrm{W}_{\mathrm{i}}\right]$. Para serem expressos em percentagem, os valores encontrados foram multiplicados por 100 .

\section{Elaboração da proposta metodológica para a previsão dos impactos em casos de rompimento de barragens de rejeito e aplicação da metodologia em barragem de mineração ativa}

Após a realização do brainstorming e a obtenção dos pesos distributivos, serão determinados os valores dos subcritérios, de acordo com a magnitude do impacto. Será estimado o valor do índice de impactos ambientais (IA) previstos para cada barragem de contenção de rejeitos da mineração por meio da aplicação da Equação 8, proposta por Carvalho (2018).
$\mathrm{IA}=\sum_{i=1}^{n} \mathrm{C}_{\mathrm{i}} \cdot \mathrm{Sc}_{\mathrm{i}}$

Em que:

$\mathrm{C}_{\mathrm{i}}=$ critério definido para avaliação de previsão de impactos;

$\mathrm{Sc}_{\mathrm{i}}=$ subcritério correspondente ao critério avaliado;

$\mathrm{n}=$ número de critérios.

Posteriormente, será elaborada uma tabela com os critérios e os subcritérios definidos no item anterior, de modo a fazer o enquadramento das barragens em relação aos aspectos apresentados. Nessa tabela, serão incluídos os pesos de cada critério e subcritério, determinados por meio do método AHP. As faixas de classificação do IA serão determinadas por meio do cálculo da média dos valores de IA encontrados para cada barragem. O valor da média somada e subtraída pelo valor do desvio padrão amostral resultará nas faixas de classificação, determinadas como baixa, média ou alta.

Finalmente, será realizado um comparativo entre a classificação proposta e as classificações determinadas pela Resolução CNRH n ${ }^{\circ}$ 143/2012, pela Portaria DNPM nº 70.389/2017 e pela DN COPAM n 87/2005. Para demonstrar a aplicação da metodologia, ela será testada em uma barragem ativa, localizada no Quadrilátero Ferrífero, Minas Gerais, por ser uma área de intensa atividade mineradora.

Tabela 3 - Índice randômico médio do Analytic Hierarchy Process.

\begin{tabular}{c|c} 
Ordem da matriz & Valores de IR \\
\hline 2 & 0,00 \\
\hline 3 & 0,58 \\
\hline 4 & 0,90 \\
\hline 5 & 1,12 \\
\hline 6 & 1,24 \\
\hline 7 & 1,32 \\
\hline 8 & 1,41 \\
\hline 9 & 1,45 \\
\hline
\end{tabular}

IR: índice randômico médio.

Fonte: Saaty (1991)

Tabela 4 - Matriz contendo os julgamentos paritários para os critérios de classificação dos impactos em casos de rompimentos de barragens de contenção de rejeitos.

\begin{tabular}{|c|c|c|c|c|c|}
\hline Linha Y & 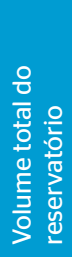 & 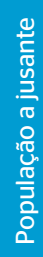 & 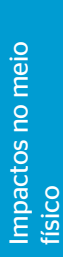 & 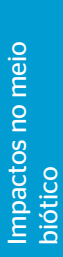 & 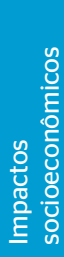 \\
\hline Volume total do reservatório & 1 & $1 / 9$ & $1 / 6$ & $1 / 6$ & $1 / 3$ \\
\hline População a jusante & 9 & 1 & 3 & 3 & 5 \\
\hline Impactos no meio físico & 6 & $1 / 3$ & 1 & 1 & 3 \\
\hline Impactos no meio biótico & 6 & $1 / 3$ & 1 & 1 & 3 \\
\hline Impactos socioeconômicos & 3 & $1 / 5$ & $1 / 3$ & $1 / 3$ & 1 \\
\hline
\end{tabular}

Fonte: Carvalho (2018). 


\section{RESULTADOS E DISCUSSÃO}

Os resultados e as discussões da metodologia aplicada neste trabalho estão descritos a seguir.

\section{Definição dos critérios}

Neste estudo, foram definidos os seguintes critérios para a aplicação do brainstorming: volume do reservatório, existência de população a jusante, impactos no meio físico, impactos no meio biótico e impactos no meio socioeconômico.

\section{Brainstorming e aplicação do método Analytic Hierarchy Process}

O brainstorming aconteceu em setembro de 2018, na Universidade Federal de Ouro Preto, onde foram comparados os julgamentos paritários dos critérios de acordo com a Tabela 1. Nos casos em que os julgamentos dos especialistas foram distintos, o moderador respeitou as diretrizes descritas na metodologia adotada por Donassollo (2017) para definição do valor. A Tabela 4 apresenta o resultado da compilação dos julgamentos.

Conforme é possível observar, os valores da tabela na diagonal principal foram equivalentes a 1. Esse resultado é esperado, pois a comparação de um critério sobre ele mesmo tem o mesmo grau de importância.

Aplicando-se os valores obtidos na Tabela 4 nas equações citadas no item anterior, para aplicação do método AHP e hierarquização dos critérios, foram encontrados valores de IC e CR equivalentes a 0,02. De acordo com Saaty (1991), os valores encontrados para IC e CR são aceitáveis e os resultados foram consistentes, pois ambos foram menores que 0,1 . Multiplicando-se cada valor do vetor peso [Wi] por 100, obteve-se os pesos percentuais (Tabela 5) de cada critério.

Conforme observado nas demais classificações de dano potencial, o critério de maior peso permaneceu sendo a presença de população na área a jusante da barragem, totalizando $47,24 \%$ da representatividade total. Em segundo lugar, ficaram empatados, com $20,31 \%$ cada, os impactos ao meio físico e os impactos ao meio biótico, isto é, os impactos sobre aspectos como qualidade da água e do solo foram tão importantes quanto os impactos sobre a flora e a fauna.

\section{Metodologia proposta para a previsão de impactos em casos de rompimentos de barragens de rejeito, comparativo entre as legislações e normas atuais e aplicação do método em barragem do Quadrilátero Ferrífero}

Com o brainstorming realizado, os critérios definidos na Figura 2 foram devidamente hierarquizados de acordo com o grau de importância a eles atribuídos. Dessa forma, a etapa seguinte consistiu na atribuição de valores para os subcritérios pré-determinados. Conforme estabelecido na elaboração da proposta metodológica, os pesos foram estimados em razão da magnitude de cada um, ou seja, para os subcritérios de menor potencialidade foram atribuídos valores iguais a 1. Para os subcritérios de maior potencialidade, isto é, de maior magnitude, foram atribuídos valores 5 .

O valor do IA para cada barragem pode ser obtido por meio do somatório dos subcritérios em que foram classificados, cada um deles multiplicados pelo peso do critério correspondente, conforme definido por Carvalho (2018) na Equação 8.

Carvalho (2018) aplicou, ainda, a metodologia em barragens de alto dano potencial do Quadrilátero Ferrífero. Posteriormente, realizou o cálculo da média dos valores de IA encontrados para cada uma delas. O valor da média, somada e subtraída pelo valor do desvio padrão amostral, resultou nas faixas de classificação apresentadas na Tabela 6 .

A Tabela 7 apresenta os critérios e os subcritérios definidos para a obtenção do IA de cada barragem, conforme descrições apresentadas na Metodologia. Para a mensuração do IA, é necessário realizar o enquadramento da barragem em relação aos aspectos apresentados. Desse modo, é necessário levantar dados a respeito do volume e do tipo de material armazenado na barragem em relação a sua periculosidade, conforme a Norma Brasileira (NBR) 10004/2004, da Associação Brasileira de Normas Técnicas (ABNT). Além disso, é necessário enquadrá-la em relação aos aspectos dos meios físicos, bióticos e socioeconômicos. Informações da estrutura e da área a jusante podem ser encontradas em processos de licenciamento e EIAs realizados para essas estruturas.

O Quadro 2 apresenta um comparativo entre a metodologia proposta e as classificações elaboradas pelas portarias, resoluções e normativas vigentes. Analisando esse quadro, é possível realizar algumas considerações. O critério "ocupação humana a jusante" foi pontuado com maior valor que os demais critérios na maioria das legislações e na metodologia proposta. Tal fato se torna coerente, principalmente, ao se observar os casos mais recentes de rompimentos de barragens de rejeito, em Minas Gerais. Quanto maior a ocupação humana a jusante, especialmente na área de autossalvamento (ZAS), maior tende a ser o número de vítimas. Vale salientar que a presença de população na área a jusante do reservatório não está apenas associada à perda de vidas, mas também a outros aspectos, como desalojamentos, redução da qualidade de vida e traumas psicológicos.

Tratando-se dos impactos ambientais, sua falta de detalhamento nas legislações e a atribuição do mesmo valor para esse critério em relação aos demais, como ocorre na DN COPAM nº 87/2005, é um aspecto que merece atenção, uma vez que, tendo sido impactados aspectos como solo e água, toda a cadeia trófica pode ser alterada e gravemente prejudicada.

Para aplicação da metodologia, selecionou-se uma barragem localizada no Quadrilátero Ferrífero. Trata-se de uma importante província geológica que ocupa uma área de $7.000 \mathrm{~km}^{2}$, localizada na porção sudeste do estado de Minas Gerais.

Tabela 5 - Pesos distributivos dos critérios para avaliação dos impactos decorrentes de rompimentos de barragens de contenção de rejeitos.

\begin{tabular}{c|c} 
Critérios & Peso distributivo (\%) \\
População a jusante & 47,24 \\
\hline Impactos diretos - Meio físico & 20,31 \\
\hline Impactos diretos - Meio biótico & 20,31 \\
\hline Impactos diretos - Meio socioeconômico & 8,47 \\
\hline Volume total do reservatório & 3,68 \\
\hline
\end{tabular}

Fonte: Carvalho (2018)

Tabela 6 - Faixas de classificação do índice de impactos ambientais para barragens consideradas de alto dano potencial.

\begin{tabular}{c|c} 
Impacto ambiental & Classificação \\
$\mid \mathrm{A}<280$ & Baixo \\
\hline $280 \leq \mid \mathrm{A}<383$ & Médio \\
\hline$I \mathrm{~A} \geq 383$ & Alto
\end{tabular}

A: índice de impactos ambientais.

Fonte: Carvalho (2018) 
Quadro 2 - Comparativo entre a metodologia proposta e as legislações e normas vigentes.

\begin{tabular}{|c|c|c|}
\hline $\begin{array}{c}\text { Resolução CNRH n } 143 / 2012 \text { e Portaria DNPM nº } \\
70.389 / 2017\end{array}$ & DN COPAM nº 87/2005 & Metodologia proposta \\
\hline \multicolumn{3}{|l|}{ Ocupação humana a jusante } \\
\hline $\begin{array}{l}\text { Critério com maior pontuação. Não considera número } \\
\text { de habitantes. }\end{array}$ & $\begin{array}{l}\text { Diferença entre a ocupação humana a jusante e os } \\
\text { demais critérios não se dá de forma tão efetiva. Não } \\
\text { considera número de habitantes. }\end{array}$ & $\begin{array}{l}\text { Critério com maior peso, sendo a diferença de } 26 \% \\
\text { para o segundo critério mais pontuado. Considera os } \\
\text { números de habitantes na região. }\end{array}$ \\
\hline \multicolumn{3}{|c|}{ Impactos ambientais - Meios físico, biótico e socioeconômico } \\
\hline $\begin{array}{l}\text { Peso menor que a ocupação humana a jusante e } \\
\text { maior que os demais. Não são considerados ou } \\
\text { detalhados os impactos à fauna e à flora, e aspectos do } \\
\text { meio físico, como qualidade dos recursos hídricos e do } \\
\text { solo na área a jusante. }\end{array}$ & $\begin{array}{l}\text { Os impactos ambientais recebem o mesmo valor de } \\
\text { importância dos demais critérios, e assim como as } \\
\text { demais legislações aqui apresentadas, não consideram } \\
\text { aspectos específicos sobre a fauna, a flora, a qualidade } \\
\text { do solo e da água. }\end{array}$ & $\begin{array}{l}\text { Impactos nos meios físico e biótico possuem o mesmo } \\
\text { valor de importância, sendo superiores aos impactos } \\
\text { do meio socioeconômico e ao volume do reservatório, } \\
\text { mas inferiores à ocupação humana a jusante. }\end{array}$ \\
\hline \multicolumn{3}{|l|}{ Volume do reservatório } \\
\hline $\begin{array}{l}\text { Critério com menor pontuação, assim como na } \\
\text { metodologia proposta. }\end{array}$ & $\begin{array}{l}\text { Critério apresenta a mesma pontuação que os } \\
\text { impactos ambientais e os impactos socioeconômicos. }\end{array}$ & Menor pontuação entre os critérios avaliados. \\
\hline
\end{tabular}

Fonte: CNRH (2012); COPAM (2005); DNPM (2017).

CNRH: Conselho Nacional de Recursos Hídricos; DNPM: Departamento Nacional de Produção Mineral; DN: Deliberação Normativa; COPAM: Conselho Estadual de Política Ambiental.

Tabela 7 - Metodologia proposta para a obtenção do índice dos impactos ambientais previstos em casos de rompimentos de barragens de contenção de rejeitos.

Critérios

Volume total do reservatório

(m3) (Portaria DNPM no

70.389/2017)

$3,68 \%$

População a jusante diretamente afetada

(habitantes)

$47,24 \%$

$47,24 \%$

Impactos ambientais diretos -

Meio físico

$20,31 \%$

Impactos ambientais diretos -

Meio biótico

$20,31 \%$

Impactos ambientais diretos -

Meio socioeconômico

$8,47 \%$

Subcritérios

1 Muito pequeno: $\leq 500 \mathrm{mil} \mathrm{m}^{3}$

2 Pequeno: 500 mil a 5 milhões $\mathrm{m}^{3}$

3 Médio: 5 a 25 milhões $m^{3}$

4 Grande: 25 a 50 milhões $\mathrm{m}^{3}$

5 Muito grande: $\geq 50$ milhões $m^{3}$

$1 \leq 100$ habitantes ou presença de pessoas temporárias ou transitando na área diretamente afetada

2101 a 5 mil habitantes

3 5.001 a 10 mil habitantes

4 10.001 a 20 mil habitantes

5 > 20 mil habitantes

1 In: Resíduos Classe II B - Inertes, segundo a ABNT NBR 10004/2004, e a área já se encontra totalmente descaracterizada de suas condições naturais.

2 PS: Resíduos Classe II B - Inertes, segundo a ABNT NBR 10004/2004. Curso d’água mais próximo é da ordem 1 ou 2, com alguma

2 alteração na qualidade da água e/ou alteração na paisagem pouco significativa.

S: Resíduos Classe II B - Inertes, segundo a ABNT NBR 10004/2004. Ocorre alteração significativa do relevo e da paisagem,

3 além da alteração da qualidade do solo e da água. Curso d'água é de ordem $\geq 3$.

MS: Resíduos Classe II A - Não Inertes, segundo a ABNT NBR 10004/2004. Ocorre descaracterização do relevo e da paisagem,

4 alteração da qualidade do solo e significativo aumento da turbidez da água. Curso d’água próximo é de ordem $\geq 3$.

5 MSA: Resíduos Classe I - Perigosos, segundo a NBR 10004 da ABNT. Ocorre total descaracterização do relevo e da paisagem,

5 significativo aumento da turbidez e contaminação da água e do solo por metais pesados. Curso d’água próximo é de ordem > 3.

1 In: Não há áreas de relevância ambiental ou protegidas por lei. Fauna e flora já bastante alteradas pela descaracterização da região.

2 Bl: Baixa diversidade e riqueza de espécies. Afugentamento das espécies da área afetada para regiões adjacentes.

3 Ml: Há diversidade e riqueza de espécies, mas algumas apresentam algum risco de extinção. Perda de indivíduos da biota pela

3 passagem do rejeito. Evasão temporária da fauna para áreas adjacentes.

4 Al: Há áreas de relevância ambiental ou protegidas por lei. Perda de habitat para a flora e a fauna, levando à evasão permanente de indivíduos, e perda das espécies.

5 AIA: Há áreas de relevância ambiental ou protegidas por lei. Perda de habitat para a flora e a fauna, levando à evasão permanente de

indivíduos, contaminação da cadeia trófica e perda de espécies.

1 I: Não existe presença de instalações residenciais, agrícolas ou de infraestrutura.

2 B: Pequena concentração de instalações residenciais, agrícolas ou de infraestrutura. Uso da água para consumo ou atividades de

2 pesca. Pouca empregabilidade.

M: Instalações residenciais, agrícolas, comerciais e estudantis. Uso da água para consumo e atividades de pesca.

3 Presença de rodovias, estradas ou ferrovias. Médio grau de empregabilidade na região.

4 A: Presença de instalações residenciais, agrícolas, comerciais e estudantis. Distribuição de água para consumo e atividades de pesca

4 Presença de indústrias, hospitais ou barragens. Alto grau de empregabilidade local.

MA: Elevada concentração de residências e instalações comerciais, estudantis e industriais. Captação de água no curso d'água

5 próximo. Presença de hospitais, barragens e mina operante. Trânsito intenso em rodovias, ferrovias e estradas. A maioria dos habitantes trabalha na cidade local.

DNPM: Departamento Nacional de Produção Mineral; ABNT: Associação Brasileira de Normas Técnicas; NBR: Norma Brasileira; In: insignificante; PS: pouco significativo; S: significativo; MS: muito significativo; MSA: muito significativo agravado; BI: baixo impacto; MI: médio impacto; Al: alto impacto; AlA: alto impacto agravado; I: inexistente; B: baixo; M: médio; A: alto; MA: muito alto.

Fonte: Carvalho (2018). 
Carvalho (2018) aplicou a metodologia na Barragem Casa de Pedra, da Companhia Siderúrgica Nacional (CSN), em razão de sua proximidade com o centro urbano de Congonhas e do patrimônio histórico mundial. A ANM (2019) classificou o barramento como de "alto dano potencial" e "baixo risco". De acordo com a DN COPAM n 87/2005, a Barragem Casa de Pedra é Classe III (alto potencial de dano ambiental). A Tabela 8 apresenta, resumidamente, as características da estrutura, conforme dados extraídos do BDA, em 2018.

A Tabela 9 apresenta a classificação da Barragem Casa de Pedra de acordo com os critérios da Tabela 7, em função dos dados coletados no EIA do empreendimento, no BDA e no estudo realizado por Pereira (2009), o qual realizou uma simulação de eventual ruptura da barragem.

O valor obtido para o IA da Barragem Casa de Pedra (392) foi classificado como "alto", de acordo com a Tabela 6. Conforme Pereira (2009), a onda de inundação decorrente da ruptura dessa estrutura se propagaria a uma grande extensão ao longo do vale no Córrego Casa de Pedra, no rio Maranhão e no rio Paraopeba. $\mathrm{O}$ autor ressalta que os rejeitos atingiriam as propriedades e as populações ribeirinhas nas cidades de Congonhas e Jeceaba, totalizando, aproximadamente, 10 mil pessoas atingidas de alguma forma pelo rompimento (Pereira, 2009). Considerando o aumento populacional no intervalo entre o estudo de Pereira (2009) e a classificação de Carvalho (2018), a Barragem Casa

Tabela 8 - Características da Barragem Casa de Pedra de acordo com o Banco de Declarações Ambientais.

\begin{tabular}{c|c|c}
$\begin{array}{c}\text { Armazenamento do } \\
\text { reservatório } \\
\text { Rejeito }\end{array}$ & $\begin{array}{c}\text { Volume final do } \\
\text { reservatório }\left(\mathrm{m}^{3}\right)\end{array}$ & Classificação do rejeito \\
\hline $\begin{array}{c}\text { Ocupação humana a } \\
\text { jusante }\end{array}$ & $\begin{array}{c}\text { Interesse ambiental a } \\
\text { jusante }\end{array}$ & $\begin{array}{c}\text { Inerte } \\
\text { Instalações na área a } \\
\text { jusante }\end{array}$ \\
\hline $\begin{array}{c}\text { Passagem de pessoas ou } \\
\text { veículos e município. }\end{array}$ & $\begin{array}{c}\text { Area de preservação } \\
\text { permanente (APP), curso } \\
\text { dágua e mata ciliar. }\end{array}$ & $\begin{array}{c}\text { Area de plantio, área de } \\
\text { pastagem, comércio, } \\
\text { escola, estrada, hospital, } \\
\text { ponte, residência e } \\
\text { ferrovia. }\end{array}$ \\
\hline
\end{tabular}

Fonte: Carvalho (2018).

Tabela 9 - Classificação dos impactos ambientais decorrentes de um rompimento hipotético da Barragem Casa de Pedra.

\begin{tabular}{|c|c|c|}
\hline Critérios & & Subcritérios \\
\hline $\begin{array}{c}\text { Volume total do reservatório (m3) (Portaria } \\
\qquad \begin{array}{c}\text { DNPM n० } 70.389 / 2017) \\
3,68 \%\end{array}\end{array}$ & 5 & $\begin{array}{c}\text { Muito grande } \\
\geq 50 \text { milhões } \mathrm{m}^{3}\end{array}$ \\
\hline $\begin{array}{l}\text { População a jusante diretamente afetada } \\
\text { (habitantes) } \\
47,24 \%\end{array}$ & 4 & 10.001 a 20 mil habitantes \\
\hline $\begin{array}{l}\text { Impactos ambientais - Meio físico } \\
\text { 20,31\% }\end{array}$ & 3 & Significativo \\
\hline $\begin{array}{l}\text { Impactos ambientais - Meio biótico } \\
\text { 20,31\% }\end{array}$ & 4 & Alto impacto \\
\hline $\begin{array}{l}\text { Impactos ambientais - Meio socioeconômico } \\
\qquad \mathbf{8 , 4 7 \%}\end{array}$ & 5 & Muito alto \\
\hline
\end{tabular}

DNPM: Departamento Nacional de Produção Mineral; IA: índice de impactos ambientais; Ci: critério definido para avaliação de previsão de impactos; SCl: subcritério correspondente ao critério avaliado.

Fonte: Carvalho (2018). de Pedra foi enquadrada na classe "10.001 a 20.000 habitantes", em relação à população a jusante.

Vale ressaltar que, embora a Barragem Casa de Pedra seja considerada de alto dano potencial, as legislações não especificam os impactos ambientais com o mesmo número de critérios que a metodologia proposta. Além disso, a barragem foi considerada de baixo risco pelas legislações, mesmo estando a pouca distância de um centro urbano e em bacia hidrográfica importante, como a do rio Paraopeba. Isso ocorre porque as análises de risco exigidas pela Lei Federal ${ }^{\circ}$ 12.334/2010 e demais legislações vigentes em segurança de barragens de mineração no Brasil, não consideram a influência das consequências ou danos para estimar a categoria de risco, classificando-os isoladamente. A categoria de risco é atualmente determinada pelo somatório dos pontos obtidos na classificação da barragem quanto às características técnicas, ao estado de conservação e ao plano de segurança de barragens.

Estudo realizado por Carvalho (2018) para a obtenção de IA em outras barragens do Quadrilátero Ferrífero consideradas de alto dano potencial e baixo risco pelas portarias e normativas, constatou que as barragens analisadas apresentaram valores de IA diferentes entre si, em função das características da área a jusante. É importante salientar que, ainda que estejam classificadas como de alto dano, as barragens não impactarão o meio ambiente da mesma forma ou com a mesma magnitude, conforme observado nos últimos casos de rompimentos em Minas Gerais. Percebe-se que os impactos tendem a variar de acordo com as características da região. Dessa forma, quanto mais específica for a classificação de previsão de impactos, mais preciso poderá ser o planejamento das ações de mitigação e minimização dos danos causados, seja em termos ambientais, seja em relação a danos à vida humana.

É importante salientar que, quando o IA da barragem for classificado como baixo ou médio, conforme a Tabela 6, isso não implica na inexistência ou insignificância dos impactos de ruptura, uma vez que todas as barragens classificadas por Carvalho (2018) são classificadas como de alto dano potencial. A classificação do IA permite realizar um comparativo entre os impactos decorrentes de cada estrutura, possibilitando distinguir entre elas quais possuem maior intensidade e quais são as maiores fragilidades da área a jusante.

\section{CONCLUSÕES}

A análise das portarias, resoluções e normativas vigentes em relação à classificação do dano potencial de barragens de rejeito, constatou a necessidade de desenvolvimento de uma proposta metodológica para determinação do IA para cada estrutura. Por meio dessa classificação, faz-se possível realizar um comparativo entre barragens consideradas de alto dano potencial e baixo risco pelas legislações vigentes - as quais se apresentam em grande número, de acordo com a classificação da ANM (2019) —, sendo possível avaliar quais barragens, quando comparadas entre si, apresentam impactos de maior ou menor intensidade e magnitude.

A utilização do AHP para a definição dos pesos de cada critério mostrou-se consistente e eficiente para a avaliação de impactos decorrentes de rupturas de barragens de rejeito. Conforme previsto, a presença de população humana a jusante foi o critério com o maior valor de importância, acompanhado pelos impactos nos meios físico e biótico. De acordo com Sánchez (2013), toda a previsão de impactos possui uma margem de incerteza associada.

A aplicação da metodologia na Barragem Casa de Pedra, da CSN, verificou a eficácia do IA. O resultado (IA alto) era esperado, considerando a proximidade com 
o centro urbano e sua inserção na Bacia do Rio Paraopeba. Dessa forma, a classificação permitiu identificar que, entre as barragens de alto dano potencial e baixo risco, a Barragem Casa de Pedra, se romper, poderá causar impactos superiores aos dos últimos rompimentos ocorridos no estado de Minas Gerais, os quais já são os maiores do mundo em relação a acidentes com barragens de rejeito.

É importante salientar que a população não é o único critério que pode elevar o valor de IA. Reservatórios de menor porte ou localizados em áreas com menor adensamento populacional também podem ter o seu IA elevado em função das condições dos meios biótico e físico, principalmente nos casos em que o material armazenado for considerado de alta periculosidade, pois esse aspecto é crucial para classificar as estruturas nos níveis mais elevados em relação aos impactos ambientais.
Diante da frequência de rompimentos no estado de Minas Gerais, nota-se a necessidade de atualização e reformulação nas legislações voltadas para a segurança de barragens em termos de dano potencial e categoria de risco. Espera-se que uma previsão mais precisa dos impactos oriundos de rompimentos de barragens possa favorecer a elaboração de um plano de ações mais efetivo na mitigação desses impactos em casos de ocorrência.

\section{CONTRIBUIÇõES DOS AUTORES}

Carvalho, G. B.: Conceituação, Curadoria de Dados, Investigação, Metodologia, Software, Escrita - Primeira Redação, Escrita - Revisão e Edição. Corteletti, R. C.: Supervisão, Validação, Visualização.

\section{REFERÊNCIAS}

AGÊNCIA NACIONAL DE MINERAÇÃO (ANM). Classificação das barragens de mineração brasileiras. Brasil: ANM, 2019. Disponivel em: http://www.anm. gov.br/assuntos/barragens/pasta-cadastro-nacional-de-barragens-de-mineracao/classificacao-oficial-anm. Acesso em: fev. 2019.

BERNARDI, J.V.E.; FOWLER, H.G.; LANDIM, P.M.B. Um estudo de impacto ambiental utilizando análises estatísticas espacial e multivariada. HOLOS Environment, v.1, n. 2, p. 162-172, 2001. https://doi.org/10.14295/holos.v1i2.1625

BRASIL. Lei Federal no 12.334, de 20 de setembro de 2010. Diário Oficial [da] República Federativa do Brasil, Brasília, 21 set. 2010. Disponível em: http://www.planalto.gov.br/ccivil_03/_ato2007-2010/2010/lei/l12334.htm. Acesso em: jul. 2018.

BRASIL. Ministério do Meio Ambiente (MMA). Conselho Nacional de Meio Ambiente (CONAMA). Resolução Conama no 1, de 23 de janeiro de 1986. Diário Oficial [da] República Federativa do Brasil, Brasília, Seção 1, p. 25482549, 17 fev. 1986

BRITO, L.T.L.; SILVA, A.S.; SRINIVASAN, V.S.; GALVÃO, C.O; GHEYI, H.R. Uso de análise multivariada na classificação das fontes hídricas subterrâneas da Bacia Hidrográfica do Salitre. Engenharia Agrícola, Jaboticabal, v. 26, n. 1, p. 36-44, 2006. https://doi.org/10.1590/S0100-69162006000100005

CARVALHO, G.B. Incidências de Impactos Decorrentes de Acidentes com Barragens de Rejeito. 207f. Dissertação (Mestrado em Geotecnia) Universidade Federal de Ouro Preto, Ouro Preto, 2018.

CONSELHO ESTADUAL DE POLITICA AMBIENTAL (COPAM). Deliberação Normativa no 62, de 17 de dezembro de 2002. Lex: Diário do Executivo Minas Gerais, 17 dez. 2002. 9 p.

CONSELHO ESTADUAL DE POLITICA AMBIENTAL (COPAM). Deliberação Normativa no 87. Lex: Diário do Executivo - Minas Gerais, 18 jun. 2005. 9 p.

CONSELHO NACIONAL DE RECURSOS HÍDRICOS (CNRH). Resolução no 143, de 10 de julho de 2012. Diário Oficial da União, Brasília, Seção 1, p. 149, 4 set. 2012.

CORTELETTI, R.C. Proposta de metodologia para análise de riscos geológico geotécnicos em ferrovias. Estudo de caso: Estrada de Ferro
Carajás (EFC). 135f. Tese (Doutorado em Geotecnia) - Universidade Federal de Ouro Preto, Ouro Preto, 2014.

COSTA, H.G. Introdução ao Método de Análise Hierárquica: Análise Multicritério no Auxílio à Decisão. Niterói: LATEC/UFF, 2002. 105 p.

DENÚBILA, L.A. Alterações ambientais associadas à mineração no municipio de São Carlos (SP), utilizando AHP e SIG. 292f. Dissertação (Mestrado em Geotecnia) - Universidade de São Paulo, São Carlos, 2013.

DEPARTAMENTO NACIONAL DE PRODUÇÃO MINERAL (DNPM) Portaria no 70.389, de 17 de maio de 2017. DNPM, 2017. Disponível em: https://www.gov.br/anm/pt-br/centrais-de-conteudo/dnpm/documentos/ portaria-dnpm-n-70389-de-17-de-maio-de-2017-seguranca-de-barragens/ view. Acesso em: mar. 2018

DONASSOLLO, A. Classificação de perigo de movimentos de massa em rodovias utilizando o método Analytic Hierarchy Process (AHP). Estudo de caso: Rodovia RS-115, Taquara - Gramado, RS. 179f. Dissertação (Mestrado em Geotecnia) - Universidade Federal de Ouro Preto, Ouro Preto, 2017.

FUNDAÇÃO ESTADUAL DO MEIO AMBIENTE (FEAM). Inventário de Barragem do Estado de Minas Gerais Ano 2015. Belo Horizonte: FEAM, 2016. 54 p

FUNDAÇÃO ESTADUAL DO MEIO AMBIENTE (FEAM). Inventário de Barragem do Estado de Minas Gerais Ano 2016. Belo Horizonte: FEAM, 2017. $47 \mathrm{p}$.

FUNDAÇÃO ESTADUAL DO MEIO AMBIENTE (FEAM). Nota de Esclarecimento 7 - Desastre Barragem B1. Belo Horizonte: FEAM, 2019 Disponível em: http://www.meioambiente.mg.gov.br/noticias/1/3745-notade-esclarecimento-7-desastre-barragem-b1. Acesso em: jan. 2019.

GIMENES, F.B.Q.; FILHO, O.A. Mapas de fragilidade ambiental utilizando o processo de análise hierárquica (AHP) e sistema de informação geográfica (SIG). In: SIMPÓSIO BRASILEIRO DE SENSORIAMENTO REMOTO, 16., 2013 Paraná. Anais [...]. Foz do Iguaçu: INPE, 2013, p. 6564-6571.

INSTITUTO BRASILEIRO DE GEOGRAFIA E ESTATISTICA (IBGE). Indicadores Sociais Municipais: uma análise dos resultados do universo do censo demográfico 2010. Rio de Janeiro: IBGE, 2011. 151 p. 
INSTITUTO BRASILEIRO DO MEIO AMBIENTE E DOS RECURSOS NATURAIS RENOVÁVEIS (IBAMA). (Org.) Laudo Técnico Preliminar: impactos ambientais decorrentes do desastre envolvendo o rompimento da barragem de Fundão, em Mariana, Minas Gerais. Brasília: IBAMA/DIPRO/ CGEMA, 2015. $38 p$

PEREIRA, F.M.S. Gestão de Risco e Plano de Ações Emergenciais Aplicado à Barragem de Contenção de Rejeitos Casa de Pedra/CSN. 159f. Dissertação
(Mestrado em Geotecnia) - Universidade Federal de Ouro Preto, Ouro Preto, 2009

SAATY, T.L. Método de Análise Hierárquica. Tradução e revisão técnica Wainer da Silveira e Silva. São Paulo: McGraw-Hill, Makron, 1991. 367 p.

SÁNCHEZ, L.E. Avaliação de Impacto Ambiental: conceitos e métodos. 2. ed. São Paulo: Oficina de Textos, 2013. 